DEFECTIVE NEUTROPHIL CHEMOTAXIS, SEVERE STAPHYLOCOCCAL INFECTION AND HYPERTMMUNOGLOBULINEMIA E. Harry R. Hil1 and Paul G. Quie. Univ. of Minnesota Medical Schoo1, Dept. of Pediatrics, Laboratory Medicine and Pathology, Minneapolis, Mn. Neutrophil granulocyte function was determined in three patients with severe staphylococcal infection, clinical manifestations of allergic disease, and hyperimmunoglobulinemia $\mathrm{E}$. Each of the patients had urticarial skin rashes before or at the time of development of staphylococcal suppurative lymphadenitis, pneumonia or sepsis. Neutrophil chemotaxis, random migration, phagocytosis and bactericidal capacity were assessed to determine if an abnormality in these functions might have contributed to the development of severe staphylococcal infection. Each of the 3 patients with increased IgE was found to have a marked defect in neutrophil chemotaxis. The mean chemotactic index of the patients was $12 \pm 4$ while that of 20 controls was $72 \pm 17$. Neutrophil random migration, phagocytosis and bactericidal capacity were normal in each patient. We have shown in previous studies that patients with eczema who suffer recurrent cutaneous staphylococcal infections may have defective neutrophil chemotaxis in association with increased IgE and have demonstrated that defective neutrophil function may result from chemical mediators such as histamine. The present patients with clinical evidence of systemic histamine release, abnormal chemotaxis and severe staphylococcal infections suggest an important relationship between allergic phenomena and the host defense mechanism.

FOLLOW-UP OF A BACTERIURIA SCREENING PROGRAM, Nancy H. Holland and Davld Goodloe, Dept. Ped., Univ, of Ky. Sch, of Med, LexIngton, Ky, and Ky. Kidney Disease Inst. (KDI), Frankfort, Ky. During 1972-73, the KDI screened 35,195 children $(17,625$ girls and 17,570 boys) for bacteriuria, defined as 3 consecutive + dipslide cultures or one + culture and + Griess nitrite test. 9,351 were preschool and 25,844 first grade children primarily. Each child's physician was requested to report results of office follow-up. Failure to file a report resulted in repeat referral to the physician. Follow-up was obtained in $90 \%$ of school children but-was less efficient in preschool children.

Prevalence rate for bacteriuria was $1.9 \%$ in girls, $0.4 \%$ in males. The relatively high rate in males reflects in part contamination in uncircumcised boys. Of 309 children seen in follow-up, urinary tract infection (UTI) was confirmed in 187 (60\%). Failure to conf1rm UTI in $40 \%$ may reflect the inefficiency of current office diagnostic methods since diagnosis of UTI was missed in 18 girls with + Griess tests.

Although UTI was confirmed by culture or urinalysis in 24 males, radiologic studies (RS) were done in only 4. Of 163 girls with confirmed UTI only 26 had RS; 8 (31\%) were abnormal and $4(16 \%)$ had ureteral reflux. As in prior reports, asymptomatic bacteriuria was associated with a high percentage of UT abnormalities when RS were done. Failure to confirm bacteriuria with urine cultures and to evaluate infected children radiologically indicate a need for basic physician education as a integral part of a screening program.

CLINICAL SPECTRUM OF NEONATAL GROUP B STREPTOCOCCAL DISEASE. Jorge B. Howard and George H. McCracken, Dept. Ped., Univ. of Texas Southwestern Med. Sch., Dallas.

Neonatal disease caused by group B beta hemolytic streptococci (GBS) has received considerable attention in recent years. A 5 year review of neonatal GBS disease was made to determine if an increase in incidence occurred and to define the spectrum of disease caused by this organism. 71 infants with GBS disease were seen from 196? through 1973: 25 infants (35\%) were encountered in 1973 alone. The incidence (per 1000 births) of GBS disease decreased from 1969 through 1972 and then significantly increased in 1973: 1.6 ('69), 1.3 ('70),1.0 ('71), 0.6 ('72) and 2.4 ('73). In contrast, the incidence of neonatal infection by Escherichia coli was unchanged during this period. Mortality rate was greater if septicemia or meningitis occurred within the first 48 hours of life (35\%) compared to disease presenting later (15\%).

Previous reports have emphasized the septicemic and meningitic forms of neonatal GBS disease. In this study 12 of 71 infants $(17 \%)$ manifested unusual or previousiy unrecognized forms of GBS infection: asymptomatic bacteremia in 4 infants, septic arthritis in 3 infants and osteomyelitis, ethmoiditis with orbital cellulitis, pneumonia with empyema, facial cellulitis, and conjunctivitis in 1 infant each. Familiarity with the diverse clinical presentations of neonatal GBS disease should increase prompt recognition and treatment as well as institution of appropriate epidemiologic controls to prevent spread of infection to personnel and other babies.
PREVENTION AND TREATMENT OF PNEUMOCYSTIS CARINII PNEUMONITIS (PCP). Walter T. Hughes, Paul C. McNabb, Thomas D. Makres, and Sandor Feldman. Infectious Diseases Service, St. Jude Children's Research Hospital, Memphis, TN.

In the immunosuppressed host the mortality rate for PCP is approximately $100 \%$ if untreated. Pentamidine isethionate is presently considered the drug of choice for therapy but has several disadvantages. The cortisone-treated rat provides an excellent model for the study of PCP. Three drugs, not previously investigated for effectiveness against $P$. carinii but with possible antiprotozoan activity, were studied. Sprague-Dawley rats were randomly assigned to 11 groups with 15 rats per group. Drugs were administered prophylactically at the initiation of immunosuppression with cortisone acetate. To evaluate therapeutic efficacy, drugs were administered after the first animal in each group had died with PCP. Lung sections taken after spontaneous death or sacrifice were examined histologically with the methenamine silver nitrate impregnation method.

Whereas, all of the 15 untreated rats died with PCP, none of the 15 given trimethoprim-sulfamethoxazole (TS) prophylactically acquired the infection. After PCP was established, 9 of 14 rats recovered after treatment with TS compared to only 2 of 14 treated with pentamidine. Rifampin and clindamycin, separately, or in combination with pentamidine, were ineffective in the prevention and treatment of PCP.

Since the animal studies clearly demonstrated that TS was effective in the prevention and treatment of PCP, we have treated to date 1 child with PCP and acute leukemia. Prompt and uncomplicated recovery followed the administration of TS orally.

\section{YERSINIA ENTEROCOLITICA ARTHRITIS}

Jerry C. Jacobs, College of Physicians and Surgeons, Columbia University, Babies Hospital, Department of Pediatrics, New York City. Introduced by Richard E. Behrman, M. D.

Human infections with Yersinia were first recognized and reported in upper New York State almost forty years ago. Little attention was paid to these reports and few efforts made to identify these organisms in the United States since that time. Recently, there have been increasing numbers of reports from Scandinavia of episodes of polyarthritis associated with Yersinia infections.

The patient to be presented is a six-month old girl transferred from an upstate New York hospital because of two weeks of fever to $104^{\circ}$, green watery diarrhea and irritability noted when she was handled. An evanescent rash was most apparent at times of temperature elevations. Her mother and father had had diarrhea during the month preceding the child's illness.

Stool cultures were grown with an effort to identifying Yersinia enterocolitica if it was present and the organism was found. Confirmation of this identification was afforded by the Plague Research Center of the United States Public Health Service where over the next month a rise in serum agglutination titer against polyvalent Y. enterocolitica was demonstrated from zero to $1: 256$ dilutions.

The clinical spectrum of disease so far known to be associated with these organisms will be discussed and the necessity for special culture techniques emphasized.

CONGENITAL MIXED CYTOMEGALOVIRUS (CMV) AND EPSTEIN-BARR VIRUS (EBV) INFECTION: REPORT OF A CASE, Jean H. Joncas, José Menezes, Maryse Granger-Julien, Bernadette McLaughlin (Intr. by Jacques R. Ducharme), From the Inst. of Microbiology and the Dept. of Microbiology and of Ped. of the Univ. of Montreal and Ste Justine Hosp., Montreal, Canada.

A 2027 gms infant was born with a petechial rash and periventricular calcifications. Total IgM was $84 \mathrm{mgs} \%$ at birth and $160 \mathrm{mgs} \%$ at age 5 months. Characteristic inclusion bodies were observed in the cells of the urinary sediment and CMV was isolated from three successive urines collected from birth to age 5 months. Peripheral leukocytes from this infant at age 4 months were established as a permanent lymphoblastoid cell line of $B$ cell characteristics. EBV or CMV antigens or virus particles could not to date be detected in these cells even following bromodeoxyuridine. Serological tests done on sera taken in the immediate neonatal period and at age 4 months revealed CMV IgM $(1 / 160)$ and $\operatorname{EBV} \operatorname{IgM}(1 / 80-1 / 20)$ antibodies by indirect immunofluorescence (Hanshaw and Schmitz and Scherer's methods respectively). A significant rise in $\mathrm{EBV}$ viral capsid antigen antibodies $(1 / 40-1 / 160)$ and CMV complement fixing antibodies (1/4-1/256) was shown. EBV early antigen antibodies were not detected $(<1 / 5)$. The establishment of a permanent lymphoblastoid cell line with $B$ cell characteristics from this infant suggests a mixed CMV-EBV infection rather than a simple serological cross reaction. 